\title{
Analytical Model for Crosstalk in p-Nwell Photodiodes
}

Beatriz Blanco-Filgueira, Paula López Martínez, Juan Bautista Roldán Aranda, and Johann Hauer

Version: accepted article

\section{How to cite:}

Beatriz Blanco-Filgueira, Paula López Martínez, Juan Bautista Roldán Aranda, and Johann Hauer (2015). Analytical Model for Crosstalk in p-Nwell Photodiodes. IEEE TRANSACTIONS ON ELECTRON DEVICES, 62(2), 580-586.

Doi: 10.1109/TED.2014.2375345

\section{Copyright information:}

(C) 2015 IEEE. Personal use of this material is permitted. Permission from IEEE must be obtained for all other uses, in any current or future media, including reprinting/republishing this material for advertising or promotional purposes, creating new collective works, for resale or redistribution to servers or lists, or reuse of any copyrighted component of this work in other works. 


\title{
Analytical Model for Crosstalk in $\mathrm{p}-\mathrm{N}_{\text {well }}$ Photodiodes
}

\author{
Beatriz Blanco-Filgueira, Paula López Martínez, Juan Bautista Roldán Aranda, and Johann Hauer
}

\begin{abstract}
The response and crosstalk (CTK) of the $\mathrm{p}-\mathrm{N}_{\text {well }}$ photodiode were studied through device simulations performed with ATLAS and experimental data. As a result, a closed-form and explicit 2D analytical model for its photoresponse and CTK was developed. The model has very few fitting parameters since it is physically-based and describes the CTK dependencies on light conditions and physical, geometrical and process parameters. This is of great interest for pixel design optimization to fulfill high resolution and small area requirements driven by pixel size reduction. As this model extends a previous one focused on $\mathbf{p}-\mathbf{n}^{+}$ devices, the behavior of both structures was also compared.
\end{abstract}

Index Terms-Crosstalk (CTK), modeling, photodiodes (PDs), simulation

\section{INTRODUCTION}

A KEY parameter to describe the performance of image sensors with ever shrinking pixels is the crosstalk (CTK). The CTK of a pixel originates due to photocarriers generated by illumination of another device in its neighborhood, which results in an unwanted output signal. Photons that penetrate the surface of a photodiode (PD) but reach another device due to a particular incident angle or the misalignment of microlenses are the source of the so-called optical CTK. Thus, this component depends on the illumination conditions and fabrication process modifications. However, what is always present in standard fabrication processes and is the focus of this work, is the electrical CTK due to diffused photocarriers from neighboring PDs. Although fabrication process modifications are frequently applied as a method of reducing both optical and electrical CTK, with these practices the advantages of using standard CMOS processes are sacrificed, [1].

In particular, CTK in CMOS image sensors also depends on the location of the pixels in the array and on the pixel layout, that is, the arrangement of the electronics and the PD within the pixel area. Some papers have also reported that CTK not only depends on the pixel geometry but also on its size, [2] [4]. Thus, as pixels shrink in order to improve the resolution of the sensor, CTK increases [5].

This work has been partially supported by the Spanish Government under projects TEC2009-12686 and TEC2012-38921-C02-02 (co-funded by the European Region Development Fund, ERDF/FEDER), by the Xunta de Galicia under project 10PXIB206037PR, by the Junta de Andalucía under project P08-TIC-3580 and by AE CITIUS under the project CN2012/151 of the Xunta de Galicia (ERDF/FEDER).

B. Blanco-Filgueira and P. López are with the Centro de Investigación en Tecnologías de la Información (CITIUS), University of Santiago de Compostela, Santiago de Compostela 15782, Spain.

J. B. Roldán is with the Department of Electronics and Computer Technology, University of Granada, Granada 18071, Spain.

J. Hauer is with the Department of IC Design - Analog Systems, Fraunhofer Institute for Integrated Circuits IIS, Erlangen 91058, Germany.
CTK study is particularly complex because its experimental characterization basically involves one of two different strategies which become more demanding as PDs shrink. The first strategy may suffer from diffraction due to the knifeedge effect, as it consists in the use of masks to block the penetration of light in some pixels to measure the response due to the illumination of unmasked pixels, [6]. It also requires the fabrication of test structures for CTK characterization expressly. The other is based on the so-called spot-scanning technique, in which an individual pixel is illuminated in order to measure its effect in the vicinity, [7]. In this case the main limitation is that it requires the use of a laser spot as small as the pixels to be characterized. For all these reasons, alternative strategies, such as the use of device simulators, become essential for the study of CTK without suffering the aforementioned practical limitations, [8], [9].

In summary, analytical modeling of CTK is desirable for pixel design optimization in order to avoid costly fabrication process modifications and characterization of test structures. Additionally, the role of analytical models in the circuit design and simulation realm is also considerable. However, there is a lack of CTK models despite their importance from the device designer viewpoint. The most remarkable approach is a semianalytical approximation in terms of the pixel geometrical shape, [3]. An analytical and more recent solution can be found in [10], but it neglects CTK to a large extent due to ad hoc modifications in a standard fabrication process.

Although CTK depends on the particular configuration of the array of pixels, the most conservative estimation can be obtained considering the worst case scenario. That is, when the PDs are close together without in-pixel electronics between them, [11]. Additionally, finding an analytical solution involves solving a $2 \mathrm{D}$ problem, as light propagation and diffusion phenomena occur in perpendicular directions.

Recently, we have reported an analytical model for CTK estimation in $\mathrm{p}-\mathrm{n}^{+}$CMOS PDs under normal incidence uniform illumination in the visible range, [12]. It is a compact, closedform, manageable expression in terms of light conditions and physical, geometrical, and process parameters validated by comparison with simulation results and experimental data. In this work, the model is extended to $\mathrm{p}-\mathrm{N}_{\text {well }}$ CMOS PDs. Section II formulates the problem and introduces the modeled structures. The development of the analytical model is explained in Section III. Section IV presents the device simulations with ATLAS and the experimental results for model validation. The crosstalk is analyzed in Section $\mathrm{V}$ in terms of geometrical parameters and illumination conditions. Finally, $\mathrm{p}-\mathrm{n}^{+}$and $\mathrm{p}-\mathrm{N}_{\mathrm{well}}$ junctions are compared in Section VI 


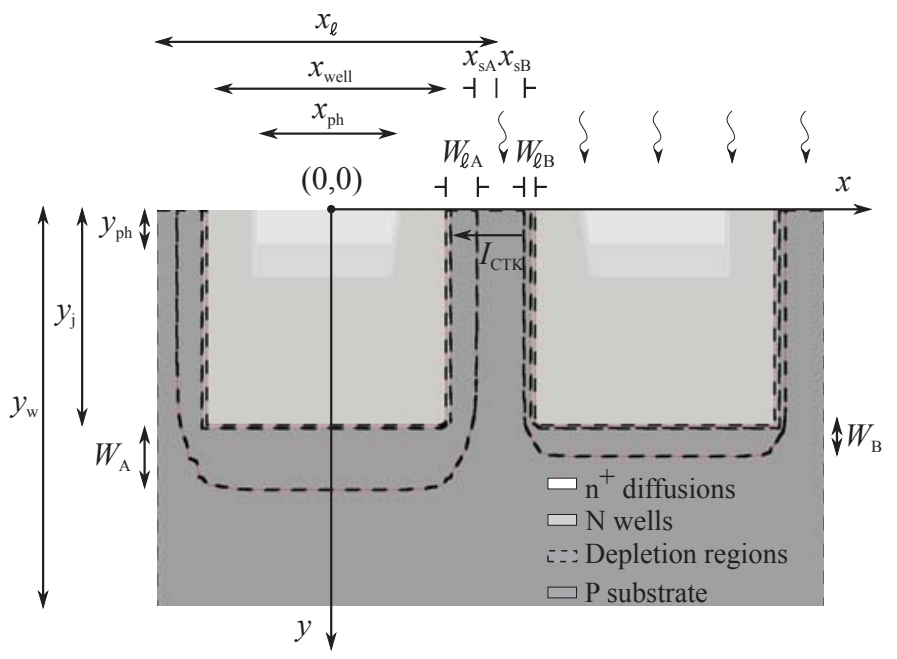

Fig. 1. Cross-section of the 3D photodiodes, A (left), and its neighbor, B (right), along with their geometrical parameters. $x_{\mathrm{SA}, \mathrm{B}}$ represent the distance between the edge of the depletion region and the limit of the photodiode for $\mathrm{A}$ and $\mathrm{B}$, respectively.

and the main conclusions are summarized in Section VII.

\section{DEVICE DESCRIPTION}

An analytical model for photoresponse and crosstalk (CTK) in $\mathrm{p}-\mathrm{n}^{+}$CMOS photodiodes has already been proposed and validated through device simulations and experimental results by the authors, [12]. In this work, we step forward introducing a new model for $\mathrm{p}-\mathrm{N}_{\mathrm{well}}$ junction photodiodes and the performance of both structures is compared.

A $\mathrm{p}-\mathrm{N}_{\text {well }}$ photodiode of small dimensions under uniform illumination in the visible range impinging perpendicularly onto the top surface is studied. For CTK analysis, two identical devices are considered close together without in-pixel electronics between them, as shown in Fig. 1. In this figure, a $2 \mathrm{D}$ cross-section of a square $\mathrm{p}-\mathrm{N}_{\text {well }}$ photodiode and its neighbor is depicted, where subscripts A (left) and B (right) represent the photodiode of interest, which is reverse-biased, and its zero-biased neighbor under illumination, respectively. The electronics in the pixel reduces CTK slightly in a real image sensor, but it depends on the particular design of the array of pixels. For this reason, the configuration chosen in this manuscript represents the worst case scenario and the most conservative estimation is calculated.

The $\mathrm{n}^{+}$diffusion, the well and the whole device are $x_{\mathrm{ph}}$, $x_{\text {well }}$ and $x_{\ell}$ wide, respectively. The device has a diffusion and junction depths $y_{\mathrm{ph}}$ and $y_{\mathrm{j}}$, respectively, and wafer thickness $y_{\mathrm{w}}$, see Fig. 1. In reverse-bias operation four main regions are distinguished: three quasi-neutral regions and the depletion region with thickness $W_{\mathrm{A}}$ (in y-direction) and $W_{\ell_{\mathrm{A}}}$ (in $\mathrm{x}$ direction). We assume the depletion region to be located in the substrate because of its lower doping concentration. The static characteristic is studied by solving the steady-state equations to describe the device transport features. The physical and illumination parameters used on subsequent sections are summarized in Table $\mathbf{I}$.
TABLE I

PARAMETERS

\begin{tabular}{|lll|}
\hline & Symbol & Unit \\
\hline Planck's constant & $h$ & $\mathrm{Js}$ \\
Speed of light & $c$ & $\mathrm{~m} / \mathrm{s}$ \\
Electron/hole lifetime & $\tau_{\mathrm{n}} / \tau_{\mathrm{p}}$ & $\mathrm{s}$ \\
Electron/hole diffusion length & $L_{\mathrm{n}} / L_{\mathrm{p}}$ & $\mathrm{m}$ \\
Absorption coefficient & $\alpha$ & $\mathrm{m}^{-1}$ \\
Transmission coefficient & $T$ & $\%$ \\
Electron/hole surface recombination velocity & $S_{\mathrm{n}} / S_{\mathrm{p}}$ & $\mathrm{m} / \mathrm{s}$ \\
Incident radiation wavelength & $\lambda$ & $\mathrm{m}$ \\
Incident optical power & $P_{\mathrm{opt}}$ & $\mathrm{W} / \mathrm{m}^{2}$ \\
Optical generation rate & $G(y)$ & $\mathrm{s}^{-1} \mathrm{~m}^{-3}$ \\
Photon flux & $\Phi(y)$ & $\mathrm{s}^{-1} \mathrm{~m}^{-2}$ \\
Photon flux at the surface & $\Phi_{0}$ & $\mathrm{~s}^{-1} \mathrm{~m}^{-2}$ \\
\hline
\end{tabular}

\section{AnAlytical MOdel}

Crosstalk (CTK) of a photodiode is commonly defined as,

$$
\operatorname{CTK}(\%)=\frac{I_{\mathrm{n}}}{I_{\text {ref }}} \times 100
$$

where $I_{\text {ref }}$ is the total photocurrent of the photodiode of interest under illumination and $I_{\mathrm{n}}$ is the photocurrent measured in the device due to the same illumination exposure of an identical neighbor. Note that both $I_{\text {ref }}$ and $I_{\mathrm{n}}$ are measured in the photodiode of interest, A in Fig. 1, which operates in the reverse-bias regime.

\section{A. Crosstalk photocurrent, $I_{n}$}

The crosstalk current due to the illumination of a neighbor photodiode, $I_{\mathrm{n}}$, is modeled as the sum of two terms: the diode A reverse-bias saturation current, $I_{0}$, and the CTK lateral current due to carriers generated in the surroundings of a zerobiased neighbor, B, under illumination, $I_{\text {СТK }}$. As p- $\mathrm{N}_{\text {well }}$ and $\mathrm{p}-\mathrm{n}^{+}$junctions have the same substrate, which is the main source of photocarriers for the crosstalk current, the analysis for the $\mathrm{p}-\mathrm{n}^{+}$can be applied to the $\mathrm{p}-\mathrm{N}_{\text {well }}$ photodiode and the details can be found in [12]:

$$
\begin{aligned}
& I_{\mathrm{CTK}}=x_{\mathrm{well}} q D_{\mathrm{n}} \sum_{n=1}^{\infty} \frac{\sqrt{\sigma_{n}}}{\theta_{n}} \\
& \quad \times\left(\frac{V_{\mathrm{B}}\left(\cosh \left(\sqrt{\sigma_{n}} x_{\mathrm{sB}}\right)-1\right)+V_{\mathrm{A}}\left(1-\cosh \left(\sqrt{\sigma_{n}} x_{\mathrm{sA}}\right)\right)}{\sinh \left(\sqrt{\sigma_{n}} x_{\mathrm{sA}}\right)}\right) \\
& \times\left(1-\cos \left(\theta_{n} y_{\mathrm{ph}}\right)\right)
\end{aligned}
$$

where

$$
\begin{gathered}
V_{\mathrm{A}}=-\frac{2(-1)^{n} \theta_{n}}{y_{\mathrm{w}}} \frac{\gamma_{\mathrm{A}} D_{\mathrm{n}}}{S_{\mathrm{n}} \sigma_{n}} \cosh \left(\frac{y_{\mathrm{w}}}{L_{\mathrm{n}}}\right) \\
V_{\mathrm{B}}=-\frac{2(-1)^{n} \theta_{n}}{y_{\mathrm{w}}}\left(\frac{\gamma_{\mathrm{B}} D_{\mathrm{n}}}{S_{\mathrm{n}} \sigma_{n}} \cosh \left(\frac{y_{\mathrm{w}}}{L_{\mathrm{n}}}\right)\right. \\
\left.+\frac{\frac{\Phi_{0} \alpha}{D_{\mathrm{n}}}}{\frac{1}{L_{\mathrm{n}}^{2}}-\alpha^{2}}\left(\frac{\sinh \left(\alpha y_{\mathrm{w}}\right)}{\alpha^{2}+\theta_{n}^{2}}-\frac{\cosh \left(\frac{y_{\mathrm{w}}}{L_{\mathrm{n}}}\right)-e^{-\alpha y_{\mathrm{w}}}}{\sigma_{n}}\right)\right)
\end{gathered}
$$

and $L_{\mathrm{n}}=\left(\tau_{\mathrm{n}} D_{\mathrm{n}}\right)^{1 / 2}, x_{\mathrm{sA}, \mathrm{B}}=x_{\ell} / 2-x_{\mathrm{well}} / 2-W_{\ell_{\mathrm{A}, \mathrm{B}}}, \theta_{n}=$ $n \pi / y_{\mathrm{w}}, \sigma_{n}=1 / L_{\mathrm{n}}^{2}+\theta_{n}^{2}$ and $n=1,2,3, \ldots S_{\mathrm{n}}, D_{\mathrm{n}}$ and $\tau_{\mathrm{n}}$ are the surface recombination velocity, the diffusion coefficient and lifetime of electrons, respectively, $\alpha$ is the absorption 
coefficient and $\Phi_{0}$ is the photon flux penetrating the silicon surface. The latter can be written as $\Phi_{0}=P_{\text {opt }} T \lambda /(h c)$, where $P_{\text {opt }}$ represents the incident optical power, $T$ the transmission coefficient, $h$ the Plank's constant, $\lambda$ the impinging radiation wavelength and $c$ the speed of light. Finally, $\gamma_{\mathrm{A}, \mathrm{B}}$ are fitting parameters. The summation in (2) is simplified because $n=1$ proved to be reasonably appropriate.

\section{B. Main photocurrent, $I_{\text {ref }}$}

With regard to the total steady-state current of the photodiode in the reverse operation regime under illumination, $I_{\text {ref }}$, it comprises the component due to the active area illumination, $I_{\mathrm{aa}}$, the photogenerated current in the lateral depletion region, $I_{\mathrm{W}}$, and the lateral photocurrent due to carriers diffused from the surroundings of the junction, $I_{\text {lateral }}$. In comparison with the results found for the $p-n^{+}$junction [12], [13], the introduction of the well modifies $I_{\text {aa }}$, as the active area has now two different components linked to the diffusion and the well, and a double junction is formed in the device.

The photocurrent due to the active area illumination, $I_{\text {aa }}$, originates from the diffusion of minority carriers in the quasi-neutral regions and drift of carriers in the depletion region due to electron-hole pairs generation. The stationary continuity equation in one dimension is solved in the quasi-neutral regions, that is, for electrons in the substrate and holes in the $\mathbf{n}^{+}$and well regions,

$$
\begin{gathered}
D_{\mathrm{n}} \frac{\partial^{2}\left(n_{\mathrm{p}}-n_{\mathrm{p} 0}\right)}{\partial y^{2}}-\frac{n_{\mathrm{p}}-n_{\mathrm{p} 0}}{\tau_{\mathrm{n}}}+G(y)=0 \\
D_{\mathrm{p}} \frac{\partial^{2}\left(p_{\mathrm{n}}-p_{\mathrm{n} 0}\right)}{\partial y^{2}}-\frac{p_{\mathrm{n}}-p_{\mathrm{n} 0}}{\tau_{\mathrm{p}}}+G(y)=0
\end{gathered}
$$

where $n_{\mathrm{p}}$ and $p_{\mathrm{n}}$ are the electron and hole concentrations and $n_{\mathrm{p} 0}$ and $p_{\mathrm{n} 0}$ their equilibrium values, respectively. $G(y)=$ $-\partial \Phi / \partial y$ is the optical generation rate, i.e. the number of photogenerated electron-hole pairs per unit volume and time. According to the Beer's law, the photon flux, $\Phi$, decreases exponentially with the depth in $\mathrm{Si}, y$, as $\Phi(y)=\Phi_{0} e^{-\alpha y}$. Equations (5) and (6) are solved subjected to the following boundary conditions

$$
\begin{aligned}
& p_{\mathrm{n}, \mathrm{d}}(0)=p_{\mathrm{n} 0}^{\text {diff }}+\left.\frac{D_{\mathrm{p}}^{\text {diff }}}{S_{\mathrm{p}}} \frac{\partial\left(p_{\mathrm{n}, \mathrm{d}}-p_{\mathrm{n} 0}^{\text {diff }}\right)}{\partial y}\right|_{y=0} \\
& p_{\mathrm{n}, \mathrm{d}}\left(y_{\mathrm{ph}}\right)-p_{\mathrm{n} 0}^{\text {diff }}=p_{\mathrm{n}, \mathrm{w}}\left(y_{\mathrm{ph}}\right)-p_{\mathrm{n} 0}^{\text {well }} \\
& -\left.q D_{\mathrm{p}}^{\text {diff }} \frac{\partial\left(p_{\mathrm{n}, \mathrm{d}}-p_{\mathrm{n} 0}^{\text {diff }}\right)}{\partial y}\right|_{y_{\mathrm{ph}}}=-\left.q D_{\mathrm{p}}^{\text {well }} \frac{\partial\left(p_{\mathrm{n}, \mathrm{w}}-p_{\mathrm{n} 0}^{\text {well }}\right)}{\partial y}\right|_{y_{\mathrm{ph}}}
\end{aligned}
$$$$
p_{\mathrm{n}, \mathrm{w}}\left(y_{\mathrm{j}}\right)=p_{\mathrm{n} 0}^{w e l l} e^{q V_{\mathrm{PD}} / K T}
$$$$
p_{\mathrm{n}, \mathrm{surr}}(0)=p_{\mathrm{n} 0}^{\text {well }}+\left.\frac{D_{\mathrm{p}}^{\text {well }}}{S_{\mathrm{p}}^{\text {well }}} \frac{\partial\left(p_{\mathrm{n}, \mathrm{surr}}-p_{\mathrm{n} 0}^{\text {well }}\right)}{\partial y}\right|_{y=0}
$$$$
p_{\mathrm{n}, \mathrm{surr}}\left(y_{\mathrm{j}}\right)=p_{\mathrm{n} 0}^{\text {well }} e^{q V_{\mathrm{PD}} / K T}
$$$$
n_{\mathrm{p}}\left(y_{\mathrm{j}}+W\right)=n_{\mathrm{p} 0} e^{q V_{\mathrm{PD}} / K T}
$$$$
n_{\mathrm{p}}\left(y_{\mathrm{w}}\right)=n_{\mathrm{p} 0}
$$

where $p_{\mathrm{n}, \mathrm{d}}, p_{\mathrm{n}, \mathrm{w}}$ and $p_{\mathrm{n} \text {,surr }}$ are the hole concentrations in the $\mathrm{n}^{+}$diffusion $\left(-x_{\mathrm{ph}} / 2 \leq x \leq x_{\mathrm{ph}} / 2\right.$ and $\left.0 \leq y \leq y_{\mathrm{ph}}\right)$, the $\mathrm{N}$ well under the diffusion $\left(-x_{\mathrm{ph}} / 2 \leq x \leq x_{\mathrm{ph}} / 2\right.$ and $\left.y_{\mathrm{ph}} \leq y \leq y_{\mathrm{j}}\right)$ and the well surrounding the diffusion $\left( \pm x_{\mathrm{ph}} / 2 \leq x \leq \pm x_{\text {well }} / 2\right.$ and $\left.0 \leq y \leq y_{\mathrm{j}}\right)$, respectively. Additionally, superscripts diff and well differentiate between the diffusion and well physical parameters. Finally, $V_{\mathrm{PD}}, K$, and $T$ are the reverse-biased voltage of the photodiode, Boltzmann constant and temperature, respectively. Regarding the carriers in the depletion region, they mainly move by drift, that is, the high electric field inside this region moves them out to the neutral regions before they can recombine. Consequently, the photogenerated current density in the depletion region can be found by integrating the generation rate, $G$, over the whole region. Finally, $I_{\mathrm{aa}}$ can be calculated as sum of drift and diffusion currents at the edges of the depletion region multiplied by the $\mathrm{n}^{+}$diffusion and surrounding well areas, $A_{\text {diff }}$ and $A_{\text {well }}$, respectively,

$$
\begin{aligned}
& I_{\text {aa }}=q A_{\text {diff }}\left(\left.D_{\mathrm{n}} \frac{\partial n_{\mathrm{p}}}{\partial y}\right|_{y_{\mathrm{j}}+W_{\mathrm{A}}}+\int_{y_{\mathrm{j}}}^{y_{\mathrm{j}}+W_{\mathrm{A}}} G(y) \mathrm{d} y-\left.D_{\mathrm{p}}^{\text {well }} \frac{\partial p_{\mathrm{n}, \mathrm{w}}}{\partial y}\right|_{y_{\mathrm{j}}}\right) \\
& +q A_{\text {well }}\left(\left.D_{\mathrm{n}} \frac{\partial n_{\mathrm{p}}}{\partial y}\right|_{y_{\mathrm{j}}+W_{\mathrm{A}}}+\int_{y_{\mathrm{j}}}^{y_{\mathrm{j}}+W_{\mathrm{A}}} G(y) \mathrm{d} y-\left.D_{\mathrm{p}}^{\text {well }} \frac{\partial p_{\mathrm{n}, \text { surr }}}{\partial y}\right|_{y_{\mathrm{j}}}\right) .
\end{aligned}
$$

Secondly, the photogenerated current in the lateral depletion region, $I_{\mathrm{W}}$, can be found by integrating the generation rate over the whole region in between the lateral junctions formed by the $s$ sides of the particular polygonal junction

$$
\begin{aligned}
I_{\mathrm{W}} & =s q \int_{-\frac{x_{\text {well }}}{2}}^{\frac{x_{\text {well }}}{2}} \int_{0}^{W_{\ell_{\mathrm{A}}}} \int_{0}^{y_{\mathrm{j}}} G(y) \mathrm{d} y \mathrm{~d} x \mathrm{~d} z \\
& =s q x_{\text {well }} W_{\ell_{\mathrm{A}}} \phi_{0}\left(1-e^{-\alpha y_{\mathrm{j}}}\right) .
\end{aligned}
$$

Finally, the lateral component due to the diffused photocarriers from the surroundings of the junction, $I_{\text {lateral }}$, is the sum of two contributions: that from those photocarriers diffused in the direction of the lateral $p-n$ junctions in the $x$ $\mathrm{z}$ plane and another from those diffused from the remaining surrounding volume, which does not form $\mathrm{p}-\mathrm{n}$ junctions with the well. The former is calculated by solving the steady-state $2 \mathrm{D}$ continuity equation because photocurrent is generated in a direction perpendicular to the illumination:

$$
\frac{\partial^{2}\left(n_{\mathrm{p}}-n_{\mathrm{p} 0}\right)}{\partial x^{2}}+\frac{\partial^{2}\left(n_{\mathrm{p}}-n_{\mathrm{p} 0}\right)}{\partial y^{2}}-\frac{n_{\mathrm{p}}-n_{\mathrm{p} 0}}{\tau_{\mathrm{n}} D_{\mathrm{n}}}+\frac{G(y)}{D_{\mathrm{n}}}=0
$$

where $\pm x_{\text {well }} / 2 \leq x \leq \pm x_{\ell} / 2$ and $0 \leq y \leq y_{\mathrm{w}}$. To solve (10) the following boundary conditions are applied:

$$
\begin{aligned}
n_{\mathrm{p}}(x, 0) & =n_{\mathrm{p} 0}+\gamma \frac{D_{\mathrm{n}}}{S_{\mathrm{n}}} \\
n_{\mathrm{p}}\left(\frac{x_{\mathrm{well}}}{2}+W_{\ell_{\mathrm{A}}}, y\right) & =0 \\
n_{\mathrm{p}}\left(x, y_{\mathrm{w}}\right) & =n_{\mathrm{p} 0} \\
n_{\mathrm{p}}\left(\frac{x_{\ell}}{2}, y\right) & =N_{\mathrm{CTK}}(y)
\end{aligned}
$$

where $\gamma$ is a fitting parameter and $N_{\text {CTK }}(y)$ stands for the excess minority carrier distribution in the border between the photodiodes due to the illumination of its neighbor, $\mathrm{B}$. Equation (10) was already solved to calculate the lateral 
current for a $\mathrm{p}-\mathrm{n}^{+}$junction and a detailed explanation can be found in [13]:

$$
I_{\text {lat }}=s \frac{2 x_{\mathrm{well}} q D_{\mathrm{n}}}{y_{\mathrm{w}}} \sum_{n=1}^{\infty} I_{1}\left(y_{\mathrm{w}}\right) I_{2}\left(x_{\mathrm{sA}}\right) I_{3}\left(y_{\mathrm{ph}}\right)
$$

where

$$
\begin{aligned}
I_{1}\left(y_{\mathrm{w}}\right) & =(-1)^{n}\left[-\frac{\gamma \frac{D_{\mathrm{n}}}{S_{\mathrm{n}}} \cosh \left(\frac{y_{\mathrm{w}}}{L_{\mathrm{n}}}\right)}{\sigma_{n}}+\right. \\
& \left.+\frac{\frac{\Phi_{0} \alpha}{D_{\mathrm{n}}}}{\frac{1}{L_{\mathrm{n}}^{2}}-\alpha^{2}}\left(\frac{\cosh \left(\frac{y_{\mathrm{w}}}{L_{\mathrm{n}}}\right)-e^{-\alpha y_{\mathrm{w}}}}{\sigma_{n}}-\frac{\sinh \left(\alpha y_{\mathrm{w}}\right)}{\alpha^{2}+\theta_{n}^{2}}\right)\right] \\
I_{2}\left(x_{\mathrm{sA}}\right) & =\frac{\sqrt{\sigma_{n}}\left(1-\cosh \left(\sqrt{\sigma_{n}} x_{\mathrm{SA}}\right)\right)}{\sinh \left(\sqrt{\sigma_{n}} x_{\mathrm{sA}}\right)} \\
I_{3}\left(y_{\mathrm{ph}}\right) & =1-\cos \left(\theta_{n} y_{\mathrm{ph}}\right)
\end{aligned}
$$

and $n=2$ in the summation proved to be appropriate. The second contribution to $I_{\text {lateral }}$ due to photocarriers in the remaining surrounding volume, which does not form $\mathrm{p}-\mathrm{n}$ junctions with the well, was estimated by device simulations. Results shown in Section IV prove that this component is not negligible and can be modeled as $I_{\mathrm{lat}} x_{\mathrm{sA}} / x_{\mathrm{well}}$, tending to match $I_{\text {lat }}$ as active area shrinks. In conclusion, lateral photocurrent is given by the sum of both components,

$$
I_{\text {lateral }}=I_{\text {lat }}\left(1+\frac{x_{\mathrm{sA}}}{x_{\mathrm{well}}}\right) \text {. }
$$

\section{EXPERIMENTAL MODEL VALIDATION}

The response of $\mathrm{p}-\mathrm{N}_{\text {well }}$ photodiodes fabricated in UMC $180 \mathrm{~nm}$ CIS (CMOS Image Sensor) technology was used in order to validate the photoresponse model. The chip consists of several sets of $3 \times 6 \mu \mathrm{m}^{2}$ 3T-APSs (Active Pixel Sensors) with photodiodes of different sizes and the same electronics in all cases. The size of the photodiodes is close to the lower limit allowed by the technology design rules. In this design, the total pixel area is divided into two equal $3 \times 3 \mu \mathrm{m}^{2}$ parts, one for the electronics (E) and another for the photodiode (PD), and the pixels are placed in a chessboard configuration, see the scheme in Fig. 2 inset. The chip also includes peripheral electronics to address the individual pixels such as a current source and row and column decoders, following the general scheme of a typical CMOS imager architecture. A picture of the experimental set-up is shown in Fig. 2. It includes a printed circuit board housing the chip, a light source, a DC voltage source to bias the chip, a signal generator to obtain the input pulses at the in-pixel electronics, logic for the column and row selection and an oscilloscope.

Characterization of test structures was performed using a $550 \mathrm{~nm}, 0.26 \mathrm{~W} / \mathrm{m}^{2}$ light source. Three pixel configurations with $x_{\ell}=3 \mu \mathrm{m}$ and different active and well areas were studied. The response of the pixels was measured in terms of sensitivity in $\mathrm{V} /(\mathrm{s} \cdot \mathrm{lux})$ during an integration time of $48 \mathrm{~ms}$. In order to find the equivalent experimental photocurrent, the sensitivity curves were reproduced by pixel simulations performed in Cadence framework. For this purpose, the transistors

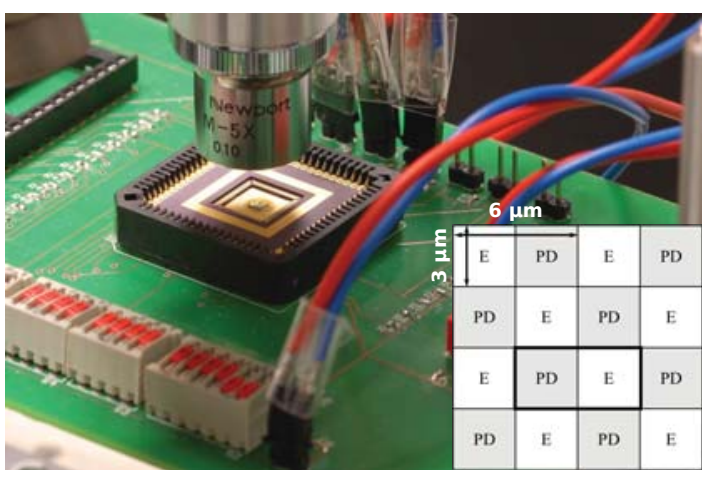

Fig. 2. Part of the experimental set-up for photodiodes characterization. The inset illustrates a schematic of the pixels arrangement which were used for $I_{\text {ref }}$ validation.

of the technology designkit which was used to fabricate the chip, UMC $180 \mathrm{~nm}$ CIS, along with the Verilog-AMS model of the photodiode, which consists of a current source associated with the intrinsic diode and the photodiode capacitance, were used. Also, a correction factor is needed to take into account the effect of the microlens over the photodiode in order to compare the experimental photocurrent with the results of the device simulations and the developed model. This factor depends on the pixel size and the photodiode area, among other parameters, and takes a value between 1.2 and 1.6 in our case for the largest and shortest junctions, respectively, which are of the same order as the values found in the literature [14]-[16].

3D numerical device simulations of the fabricated structures using ATLAS from Silvaco were also performed. The technological parameters are estimated for the $180 \mathrm{~nm}$ technological node. A pair of identical $\mathrm{p}-\mathrm{N}_{\text {well }}$ junction photodiodes was simulated under uniform illumination in the visible range impinging perpendicularly onto the top surface. Thus, $I_{\text {ref }}$ is obtained by illumination of reversebiased device $\mathrm{A}\left(-x_{\ell} / 2 \leq x, z \leq x_{\ell} / 2\right)$ whereas photodiode B $\left(x_{\ell} / 2 \leq x \leq 3 x_{\ell} / 2,-x_{\ell} / 2 \leq z \leq x_{\ell} / 2\right)$ is zero-biased, see Fig. 1 .

The response of the same photodiodes is also predicted according to the model developed in the previous section. The experimental, simulated and modeled main photocurrent, $I_{\text {ref, for }} \mathrm{p}-\mathrm{N}_{\text {well }}$ junctions with different $x_{\mathrm{sA}}$ is plotted in Fig. 3. Both the simulation results and the proposed model fit the experimental measurements with reasonable accuracy, specially for those photodiodes with small active area.

In Fig. 4, the simulated and modeled results of the different components of the main photocurrent, $I_{\text {ref }}$, are depicted. The model fits the simulated data with high precision and a minor disagreement of the active area contribution for photodiodes with a wide well. As mentioned in the previous section, the lateral contribution from photocarriers generated in the surrounding substrate, which does not form lateral p-n junctions, is not negligible and even matches the lateral component due to the p-n junctions for photodiodes with small active area.

The disagreement in the $I_{\text {aa }}$ calculation at lowest $x_{\mathrm{sA}}$ values does not compromise the model usefulness because it coincides with the limit imposed by the technology itself, which does not allow lower $x_{\mathrm{sA}}$ values. Additionally, $I_{\mathbf{n}}$ 


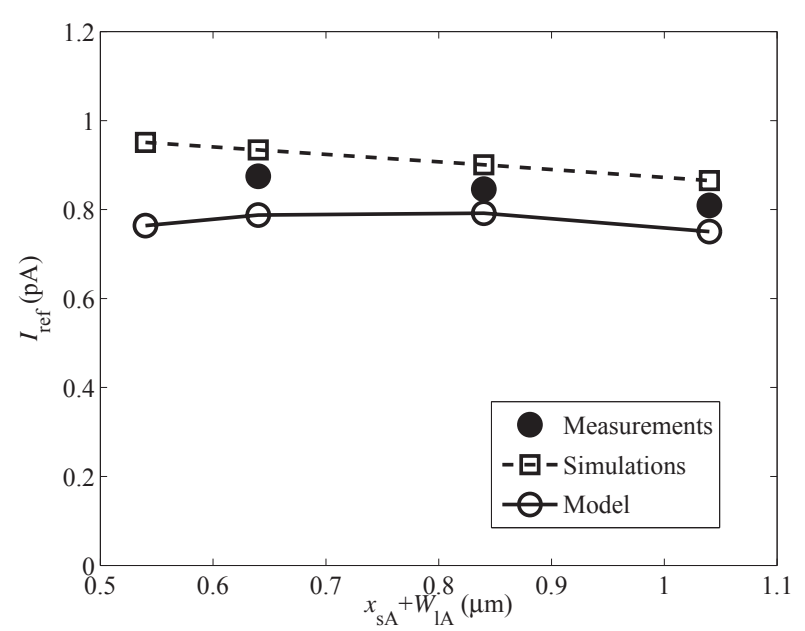

Fig. 3. Comparison of the main photocurrent, $I_{\text {ref }}$, between measured, simulated and modeled p- $\mathrm{N}_{\text {well }}$ junctions with $x_{\ell}=3 \mu \mathrm{m}$ and different $x_{\mathrm{sA}}$ values in a $180 \mathrm{~nm}$ CMOS technology under a $550 \mathrm{~nm}$ light source.

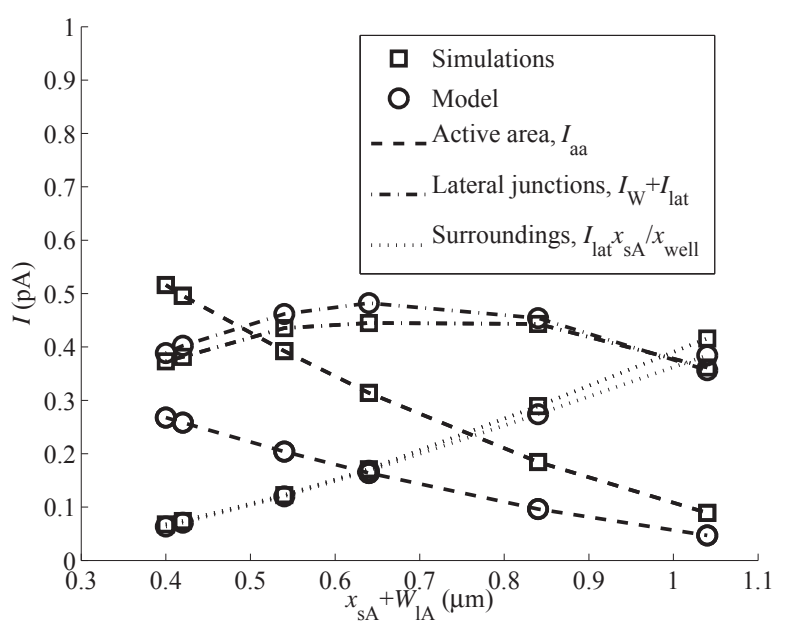

Fig. 4. Comparison between the simulated and modeled components of the main photocurrent, $I_{\mathrm{ref}}$, for $\mathrm{p}-\mathrm{N}_{\mathrm{well}}$ junctions with different $x_{\mathrm{sA}}$ in a $180 \mathrm{~nm}$ CMOS technology under a $550 \mathrm{~nm}$ light source.

results in Section $\mathrm{V}$ show that its magnitude is of the same order as $I_{\text {ref }}$ and its dependence on $x_{\mathrm{sA}}$ is stronger. Thus, the main criteria when designing an array of photodiodes should be try to minimize $I_{\mathbf{n}}$.

Summarizing, the model constitutes a powerful tool for photoresponse prediction of $\mathrm{p}-\mathrm{N}_{\text {well }}$ junctions and circuit simulations involving these devices, as the model can be easily implemented in hardware description languages.

\section{Crosstalk analysis}

The CTK given by the model was validated through $3 \mathrm{D}$ numerical device simulations using ATLAS. A pair of identical structures was simulated under uniform illumination in the visible range impinging perpendicularly onto the top surface. Thus, the main photocurrent, $I_{\text {ref }}$, is obtained by illumination of photodiode A, as explained in Section IV, and the crosstalk component, $I_{\mathrm{n}}$, is obtained by illumination of zero-biased
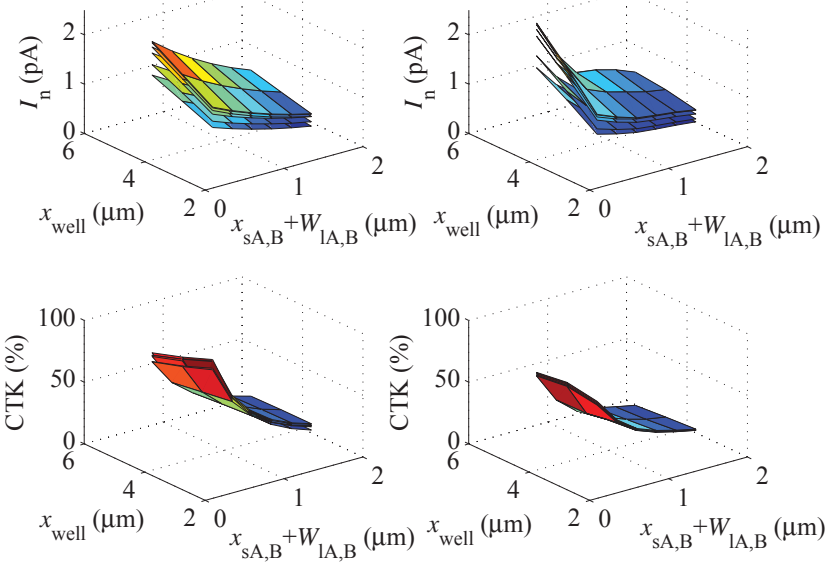

Fig. 5. Comparison between modeled and simulated results for $\mathrm{p}-\mathrm{N}_{\mathrm{well}}$ photodiodes of different dimensions in the visible range.

photodiode $\mathrm{B}\left(x_{\ell} / 2 \leq x \leq 3 x_{\ell} / 2,-x_{\ell} / 2 \leq z \leq x_{\ell} / 2\right)$, see Fig. 1. Photodiodes with different values for $x_{\mathrm{ph}}, x_{\mathrm{well}}$ and $x_{\ell}$ where chosen and the technological parameters are estimated for the $180 \mathrm{~nm}$ technological node.

Simulation results validate the analytical models for $I_{\mathrm{n}}$ and CTK. As an example, Fig. 5 depicts modeled and simulated current and crosstalk for small photodiodes with $x_{\text {well }}=$ $3.06,4.06,5.06 \mu \mathrm{m}$ and $x_{\ell}=3.56$ to $6.81 \mu \mathrm{m}$ under a $\mathrm{P}_{\mathrm{opt}}=0.26 \mathrm{~W} / \mathrm{m}^{2}$ light source. Each surface plot corresponds to a different value of $\lambda$, covering the visible range. Simulation and modeled results show a good agreement, revealing a response dependence on both the active and total photodiode areas, as previously observed for $\mathrm{p}-\mathrm{n}^{+}$junctions, [12], [13], [17]. $I_{\mathrm{n}}$ increases as well regions grow and approach each other, and when the area surrounding the junction becomes smaller. As a result in terms of CTK, the influence of the distance between the edge of the depletion region and the limit of the photodiode, $x_{\mathrm{SA}, \mathrm{B}}$, proves to be more important than the dependence on the well size, $x_{\text {well }}$, favoring CTK as the surface area surrounding the junction decreases.

The spectral response within the visible range was also studied, see Fig. 6 . In this figure, $I_{\mathrm{n}}$ and CTK curves obtained with the model and the simulator are represented in lines and circles, respectively. Photodiodes with a well width, $x_{\text {well }}$, of $3.06 \mu \mathrm{m}$ (left column), $4.06 \mu \mathrm{m}$ (middle column) and $5.06 \mu \mathrm{m}$ (right column) are compared. For each photodiode, different values of the distance between the edge of the depletion region and the limit of the photodiode, $x_{\mathrm{sA}, \mathrm{B}}$, were considered. Note that the arrow indicates the increasing direction of these parameters. Neither $\lambda$ nor $x_{\text {well }}$ present a significant effect on CTK for the considered structures. What is decisive, as it was seen previously, is the distance between the edge of the depletion region and the limit of the photodiode, $x_{\mathrm{sA}, \mathrm{B}}$.

This kind of photodiode characterization is essential from the point of view of the image sensor designer. The accurate knowledge of the dependence of CTK on the geometrical features of the structure can be used to maximize the photodiode response whereas keeping an acceptable level of CTK. It is 

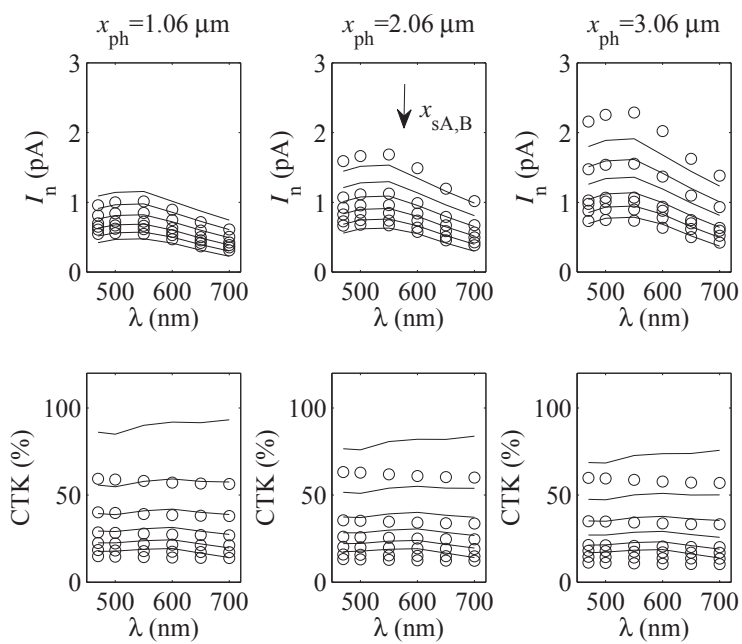

Fig. 6. Spectral response given by the model (lines) and device simulations (circles) for $\mathrm{p}-\mathrm{N}_{\text {well }}$ photodiodes with $x_{\mathrm{well}}=3.06,4.06,5.06 \mu \mathrm{m}$ and $x_{\mathrm{sA}, \mathrm{B}}+W_{\ell_{\mathrm{A}, \mathrm{B}}}=0.50,0.75,1.00,1.25,1.50,1.75 \mu \mathrm{m}$.

important to note that this is an approximation to the maximum expected CTK, that is, the worst case scenario consisting of two photodiodes without extra neighbors. However, in a real array implementation the rest of neighbors in the vicinity and the in-pixel electronics are expected to reduce CTK slightly.

\section{COMPARISON OF P-N ${ }^{+}$AND P-N $\mathrm{NELL}_{\text {PHOTODIODES }}$}

Both $\mathrm{p}-\mathrm{n}^{+}$and $\mathrm{p}-\mathrm{N}_{\mathrm{well}}$ junction photodiodes were studied through device simulations using ATLAS, were experimentally characterized and an analytical description of their photoresponse and CTK was proposed and validated. Finally, the response of both structures will be compared.

Structures with the same total width in a standard $180 \mathrm{~nm}$ technology are simulated under uniform illumination both within and beyond the limits of the visible range. A p$\mathrm{N}_{\text {well }}$ is compared with a $\mathrm{p}-\mathrm{n}^{+}$with a diffusion of the same size of the well. It can be proved that varying the diffusion width in a $p-\mathrm{N}_{\text {well }}$ without well variations results in the same response. Fig. 7 depicts example results for the response of both structures with $x_{\ell}=5.06 \mu \mathrm{m}$ and $x_{\text {well }}=3.06 \mu \mathrm{m}$ (value of $x_{\mathrm{ph}}$ for the $\left.\mathrm{p}-\mathrm{n}^{+}\right)$. The main and crosstalk photocurrents, $I_{\text {ref }}$ and $I_{\mathrm{n}}$, of the $\mathrm{p}-\mathrm{N}_{\text {well }}$ are higher than those for the $\mathrm{p}-\mathrm{n}^{+}$, specially in the visible range. Its maximum response is also located for a longer wavelength than that for $\mathrm{p}-\mathrm{n}^{+}$photodiode. In terms of CTK, the later presents a slightly better response below $\lambda=700 \mathrm{~nm}$, where the tendency is inverted, being almost the same for both structures for longer wavelengths.

\section{CONCLUSIONS}

A closed-form and explicit 2D analytical model for photoresponse and CTK estimation of CMOS $\mathrm{p}-\mathrm{N}_{\text {well }}$ photodiodes was developed and validated through device simulations and experimental measurements. It is physically-based and describes the photodiode behavior taken into consideration the light conditions and physical, geometrical and process parameters. The model constitutes a powerful tool which can be used in computer aided design tools for CMOS imagers design.
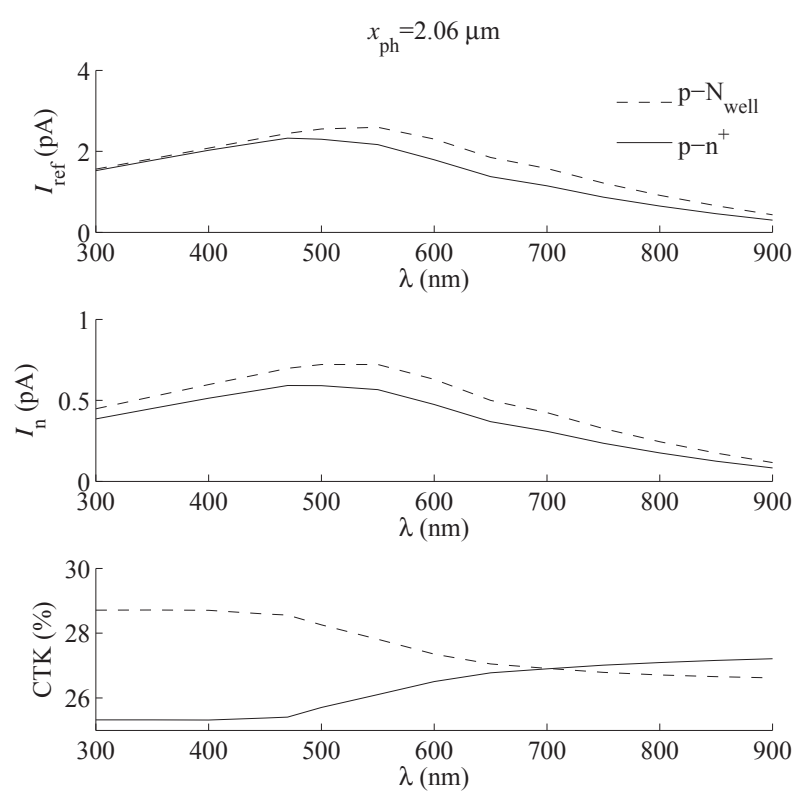

Fig. 7. Comparison of $\mathrm{p}-\mathrm{n}^{+}$and $\mathrm{p}-\mathrm{N}_{\mathrm{well}}$ photodiodes with the same total width, $x_{\ell}=5.06 \mu \mathrm{m}$, and diffusion and well areas, respectively.

\section{REFERENCES}

[1] J. Ahn et al., "A 1/4-inch 8Mpixel CMOS Image Sensor with 3D Backside-Illuminated $1.12 \mu \mathrm{m}$ Pixel with Front-Side Deep-Trench Isolation and Vertical Transfer Gate," in IEEE ISSCC Tech. Dig., Feb. 2014, pp. $124-125$.

[2] I. Shcherback and O. Yadid-Pecht, "Photoresponse analysis and pixel shape optimization for CMOS active pixel sensors," IEEE Trans. Electron Devices, vol. 50, no. 1, pp. 12-18, Jan. 2003.

[3] I. Shcherback, T. Danov, and O. Yadid-Pecht, "A comprehensive CMOS APS crosstalk study: photoresponse model, technology, and design trends," IEEE Trans. Electron Devices, vol. 51, no. 12, pp. 2033-2041, Dec. 2004.

[4] L. Blockstein and O. Yadid-Pecht, "Crosstalk quantification, analysis, and trends in CMOS image sensors," Applied optics, vol. 49, no. 24, pp. 4483-4488, Aug. 2010.

[5] C. Tseng et al., "Crosstalk Improvement Technology Applicable to $0.14 \mu \mathrm{m}$ CMOS Image Sensor," in IEEE IEDM Tech. Dig., Dec. 2004, pp. $997-1000$.

[6] M. Estribeau and P. Magnan, "Pixel Crosstalk and Correlation with Modulation Transfer Function of CMOS Image Sensor," in Proc. SPIE vol. 5677, Mar. 2005, pp. 98-108.

[7] J. S. Lee, J. Shah, M. E. Jernigan, and R. I. Hornsey, "Characterization and Deblurring of Lateral Crosstalk in CMOS image sensors," IEEE Trans. Electron Devices, vol. 50, no. 12, pp. 2361-2368, Dec. 2003.

[8] H. Mutoh, "3-D Optical and Electrical Simulation for CMOS Image Sensors," IEEE Trans. Electron Devices, vol. 50, no. 1, pp. 19-25, Jan. 2003.

[9] F. Zhang, J. Zhang, C. Yang, and X. Zhang, "Performance Simulation and Architecture Optimization for CMOS Image Sensor Pixels Scaling Down to $1.0 \mu \mathrm{m}$," IEEE Trans. Electron Devices, vol. 57, no. 4, pp. 788-794, Apr. 2010.

[10] I. Djite, M. Estribeau, P. Magnan, G. Rolland, S. Petit, and O. SaintPé, "Theoretical Models of Modulation Transfer Function, Quantum Efficiency, and Crosstalk for CCD and CMOS Image Sensors," IEEE Trans. Electron Devices, vol. 59, no. 3, pp. 729-737, Mar. 2012.

[11] I. Brouk, Y. Nemirovsky, S. Lachowicz, E. Gluszak, S. Hinckley, and E. K., "Characterization of crosstalk between CMOS photodiodes," Solid-State Electronics, vol. 46, no. 1, pp. 53-59, Jan. 2002.

[12] B. Blanco-Filgueira, P. López, and J. B. Roldán, "Closed-Form and Explicit Analytical Model for Crosstalk in CMOS Photodiodes," IEEE Trans. Electron Devices, vol. 60, no. 10, pp. 3459-3464, Aug. 2013.

[13] B. Blanco-Filgueira, P. López, and J. Roldán, "Analytical modelling of size effects on the lateral photoresponse of CMOS photodiodes," SolidState Electronics, vol. 73, no. 0, pp. 15-20, Jul. 2012. 
[14] S.-G. Wuu et al., "A high performance active pixel sensor with 0.18 $\mu \mathrm{m}$ CMOS color imager technology," in IEEE IEDM Tech. Dig., Dec. 2001, pp. 24.3.1-24.3.4.

[15] M. Cohen et al., "Fully Optimized $\mathrm{Cu}$ based process with dedicated cavity etch for $1.75 \mu \mathrm{m}$ and $1.45 \mu \mathrm{m}$ pixel pitch CMOS Image Sensors," in IEEE IEDM, Dec. 2006, pp. 1-4.

[16] S. Vargas-Sierra, E. Roca, and G. Liñán-Cembrano, "APS design alternatives in $0.18 \mu \mathrm{m}$ CMOS image sensor technology," in Proc. IEEE ECCTD, Aug. 2009, pp. 1-4.

[17] B. Blanco-Filgueira, P. López, and J. B. Roldán, “Experimental characterization of peripheral photocurrent in CMOS photodiodes down to 65 nm technology," Semicond. Sci. Technol., vol. 28, no. 4, pp. $045011-$ 045 017, Apr. 2013.

Beatriz Blanco-Filgueira received the Ph.D. degree from the University of Santiago de Compostela, Santiago de Compostela, Spain, in 2012.

She is currently a Post-Doctoral Researcher with CiTIUS, Galiza, Spain.

Paula López Martínez received the Ph.D. degree from the University of Santiago de Compostela, Santiago de Compostela, Spain, in 2003.

She is currently an Associate Professor with the University of Santiago de Compostela.

Juan Bautista Roldán Aranda received the Ph.D. degree from the University of Granada, Granada, Spain, in 1997.

$\mathrm{He}$ is currently an Associate Professor with the University of Granada.

Johann Hauer received the degree in Electrical Engineering from the University of Erlangen-Nürnberg, Germany, in 1984.

$\mathrm{He}$ is currently with the Department of IC Design-Analog Systems of the Fraunhofer Institute for Integrated Circuits, leading a group for mixed signal IC design since 1992. 